\title{
Resistencia al choque térmico de Y-TZP con fisuras Palmqvist
}

\author{
G. FARGAS RIBAS, D. CASELLAS PADRÓ, L. M. LLANES PITARCH, M. ANGLADA GOMILA
}

Departamento de Ciencia de Materiales e Ingeniería Metalúrgica. Universidad Politécnica de Cataluña, E.T.S.E.I.B, Barcelona

Se analiza el comportamiento frente al choque térmico de policristales de circona tetragonal dopados con $2.5 \%$ molar de itria (Y-TZP), con dos niveles distintos de tenacidad y con fisuras de indentación del tipo Palmqvist. El proceso de extensión de la fisura se cuantifica suponiendo que las tensiones residuales responsables de su propagación estable durante el choque térmico son inferiores a las presentes en el material inmediatamente después de aplicar la indentación. Se propone la utilización de un parámetro de resistencia al choque térmico que solamente depende de la extensión de las fisuras por efecto de las tensiones térmicas generadas durante el cambio brusco de temperatura. Los resultados del análisis se aplican al estudio del choque térmico y se comparan con los resultados experimentales.

Palabras clave: Policristales de circona tetragonal, fisuras Palmqvist, resistencia al choque térmico.

\section{Thermal shock resistance of Y-TZP with Palmqvist craks}

The thermal shock response of two tetragonal zirconia polycrystals stabilised with $2.5 \%$ molar yttria, with two different levels of fracture toughness, has been investigated. Palmqvist cracks are generated by indentation and the crack extension is quantified by assuming that the residual thermal stresses responsible for its extension during thermal shock are smaller than those acting on the crack immediately after indentation. It is proposed to use a thermal shock resistance parameter that only depends on the crack extension induced by thermal stresses generated during the abrupt changes in temperature. The results are applied to the study of thermal shock resistance and they are compared with experimental results.

Keywords: Tetragonal zirconia polycrystals, Palmqvist cracks, thermal shock resistance.

\section{INTRODUCCIÓN}

Desde hace tiempo existe un gran interés en la utilización de materiales cerámicos en aplicaciones estructurales a alta temperatura y en presencia de agentes corrosivos. Así, en las últimas décadas, se han desarrollado materiales cerámicos con tenacidad superior a la tenacidad típica de las cerámicas tradicionales, con excelentes propiedades a altas temperaturas, elevada resistencia al ataque químico, y que plantean su posible aplicación como elementos estructurales (1). Sin embargo, una de las propiedades que limitan todavía su aplicabilidad es su baja resistencia al choque térmico.

Cuando la superficie de un material cerámico es sometida a un cambio brusco de temperatura, se producen tensiones que pueden llegar a provocar la rotura del mismo. Éstas se originan debido a dilataciones diferenciales inducidas durante la aplicación de un gradiente de temperatura. En materiales policristalinos o multifásicos también pueden formarse por dilataciones diferenciales entre fases o granos vecinos (2). La sensibilidad del material a estas deformaciones determina su resistencia frente a cambios bruscos de temperatura. Por consiguiente, el comportamiento frente al choque térmico de un material depende tanto de sus propiedades mecánicas, como de sus propiedades térmicas.

Cuando las tensiones originadas durante un choque térmico son suficientemente elevadas, las fisuras existentes en el material se pueden propagar. Si la propagación es estable, no se produce la rotura total, pero sí su degradación, lo cual se puede poner de manifiesto mediante la posterior medida de diversas propiedades mecánicas. Así la caída del valor del módulo de rotura es una de las evidencias más claras que permite medir el grado de deterioro inducido por el choque térmico

Para evaluar las tensiones inducidas por el choque térmico es necesario considerar un gran número de parámetros tales como: el módulo de Young, el coeficiente de Poisson, el coeficiente de expansión térmico, la conductividad térmica del material, el tamaño y forma de la muestra y los que se refieren a características del temple como son el gradiente de temperatura y el coeficiente de transferencia de calor del medio dónde se produce (3).

Existen diversos parámetros desarrollados para la evaluación del choque térmico de materiales cerámicos, muchos de ellos introducidos por Hasselman (4,5). Recientemente, Tancret y Ostertock (6) han propuesto la utilización de uno de estos parámetros para el estudio de materiales cerámicos con fisuras semicirculares creadas por indentación. La principal ventaja de utilizar fisuras de indentación es que, a diferencia de otros métodos en los que es necesario el conocimiento de un gran número de parámetros, para la evaluación de la resistencia al choque térmico se requiere solamente medir la longitud de las 
fisuras antes y después del cambio de temperatura.

Uno de los parámetros propuestos por Hasselman es $R_{m^{\prime}}$ el cual se define como el cuadrado del cociente entre la tenacidad de fractura, $K_{c}$ y la tensión térmica, $\sigma$, que actúa sobre la superficie del material:

$$
R_{m}=\left(\frac{K_{c}}{\sigma}\right)^{2}
$$

En este trabajo se utiliza un sistema de grietas de indentación tipo Palmqvist para el estudio del choque térmico, ya que éste es el tipo de fisuras que se producen en diversos materiales de interés como, por ejemplo, la Y-TZP y los carburos cementados. A diferencia de la gran mayoría de trabajos de indentación que consideran la tensión residual constante, en el presente trabajo se permite que la tensión residual de indentación puede disminuir, ya sea por relajación de tensiones a alta temperatura, bien por la influencia de las tensiones inducidas durante el choque térmico. Finalmente, se analizan los resultados en términos de un nuevo parámetro $R$ que representa el cociente entre la tenacidad de fractura del material y el factor de intensidad de tensiones inducido sobre una fisura de indentación.

\section{FISURAS DE INDENTACIÓN TIPO PALMQVIST}

En este trabajo se estudia el comportamiento frente al choque térmico de una Y-TZP de grano fino. Dicho material desarrolla fisuras tipo Palmqvist al aplicar un penetrador Vickers. Este sistema de fisuras se forma en materiales cerámicos con tenacidad relativamente alta, como la Y-TZP y los carburos cementados. Por ello se desarrollan parámetros de resistencia al choque térmico que tengan en consideración este tipo de fisuras. Al aplicar un penetrador Vickers o Knoop sobre la superficie de un material cerámico, se forma una huella y dos sistemas de fisuras bien definidas que emanan de los vértices de la misma. Cada sistema puede estar conectado por debajo de la zona deformada y producir un perfil semicircular (Fig.1). En cerámicas con tenacidad elevada, a menudo las fisuras no están conectadas y cada sistema está formado por dos fisuras independientes comúnmente denominadas fisu-

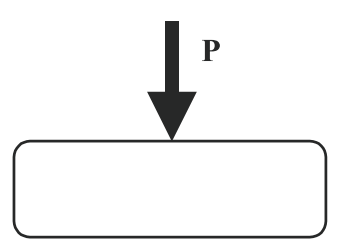

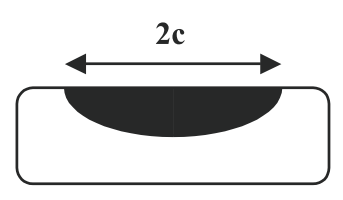

Fisuras semielípticas

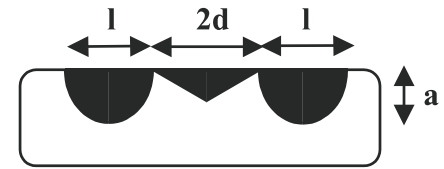

Fisuras Palmqvist
Figura 1. Forma que presentan las fisuras, una vez retirada la carga, en función del tipo de material considerado. ras Palmqvist (7)

La aplicación de una carga $\mathrm{P}$ induce tensiones residuales que son las responsables de la extensión de la fisura durante el proceso de indentación. Su longitud viene determinada por la tenacidad del material y por la carga de indentación. Por consiguiente, la medida de la longitud de las fisuras permite determinar $\mathrm{K}$ si se conoce el factor de intensidad de tensiones inducido por las tensiones residuales, $\mathrm{K}_{\mathrm{res}}$. Para fisuras tipo Palmqvist, Nihihara propuso que $\mathrm{K}_{\mathrm{res}}$ es inversamente proporcional a la raíz cuadrada de la longitud de la fisura (8):

$$
\mathrm{K}_{r e s}=\chi\left(\frac{P}{d l^{1 / 2}}\right)
$$

donde $\chi$ es una constante, $\mathrm{P}$ la carga de indentación, d la semi-diagonal de la huella de indentación y 1 la longitud de fisura. La tenacidad de fractura puede entonces determinarse a partir de la medida de la longitud de la fisura, $1_{0}(9)$ :

$$
\mathrm{K}_{c}=\chi_{0}\left(\frac{P}{d l_{0}^{1 / 2}}\right)
$$

donde $\chi_{0}$ es una constante que determina la magnitud de las tensiones residuales después de indentar cuando no hay carga externa aplicada, y $l_{0}$ es la longitud de la fisura inicial. En realidad esta circunstancia es la que permite que la fisura de indentación se propague de forma estable hasta que el factor de intensidad de tensiones residual es igual a la tenacidad de fractura del material. Si sobre la fisura actúa una fuerza externa, también en modo I, el factor de intensidad de tensiones total se obtiene sumando los factores de intensidad de tensiones de la fuerza aplicada, $\mathrm{K}_{\mathrm{ap}}$, con el correspondiente $\mathrm{a}$ las tensiones residuales. $\mathrm{El} \mathrm{K}$ total viene dado por:

$$
K=K_{r e s}+K_{a p}
$$

Para una grieta semielíptica, tanto si la tensión aplicada es constante (ensayo de tracción), o diminuye proporcionalmente con la distancia a la superficie (ensayo de flexión), se conoce que el factor de intensidad de tensiones aplicado se puede escribir como el producto de la tensión máxima aplicada, $\sigma$, por la raíz cuadrada de la longitud de la grieta y un factor geométrico $Y$, el cual ha sido determinado por Newman y Raju (10). En el caso presente, si se produce una disminución brusca de la temperatura, $\Delta \mathrm{T}$, en la superficie, la tensión alcanzará un valor máximo $\left(\sigma_{\max }\right)$ en ésta, y disminuirá hacia el interior de acuerdo a una ley del tipo:

$$
\sigma=\sigma_{\max }\left[1-\operatorname{erf}\left(\frac{x}{x_{0}} \frac{\sqrt{t_{0}}}{\sqrt{t}}\right)\right]
$$

siendo $\mathrm{x}$ la profundidad, t el tiempo, $\mathrm{y}$

$$
\sigma_{\max }=\frac{E \alpha \Delta T}{1-v}
$$

Para simplificar el cálculo supondremos que cuando las grietas se extienden mantienen constante su forma, es decir, a / l =constante; por consiguiente el valor de Y no cambia con la extensión de la grieta. En realidad la gran diferencia entre la tensión en la superficie y en el interior, provocará la extensión estable de la fisura a lo largo del semi-eje 1 antes que se alcance la condición de crecimiento en la dirección de la profundi- 
dad. Por consiguiente el perfil de la fisura tenderá a cambiar de forma. Por este motivo, aún en el caso de que no existan tensiones residuales, se puede originar cierta extensión estable de la fisura en la dirección 1. En la Fig.2 se representan de forma esquemática los dos términos de la ecuación [4] en función de la longitud de la fisura. Se puede apreciar que K disminuye al propagarse la fisura hasta llegar a un valor mínimo a partir del cual $\mathrm{K}$ aumenta.

La condición de rotura inestable se alcanza cuando: i) $\mathrm{K}$ es igual a la tenacidad de fractura del material $(\mathrm{K}=\mathrm{K})$; ii) $\mathrm{K}$ alcanza el valor mínimo (en el caso en que $K_{c}$ sea independiente de la longitud de la grieta), en caso contrario, si existe curva R no saturada entonces la pendiente de $\mathrm{K}$ debe ser igual a la pendiente de $\mathrm{K}_{\mathrm{c}^{\prime}}$ es decir:

$$
\frac{\partial K}{\partial c}=\frac{\partial K_{c}}{\partial c}
$$

\section{CHOQUE TÉRMICO EN MATERIALES CON FISURAS TIPO PALMQVIST}

Cuando una fisura de tipo Palmqvist, creada mediante la aplicación de una carga $P$, es sometida a una tensión $\sigma$, tal como ocurre durante el proceso de choque térmico, el factor de intensidad de tensiones total definido en la ecuación [4]:

$$
\mathrm{K}=\frac{\chi P}{d \sqrt{l}}+\mathrm{Y} \sigma \sqrt{l}
$$

Si inicialmente $\mathrm{K}_{\mathrm{res}}=\mathrm{K}_{c^{\prime}}$ al aplicar una tensión infinitesimal, $\mathrm{K}$ debiera ser siempre mayor que $K_{c}$ y la fisura se debería extender inmediatamente de forma estable hasta que de nuevo $\mathrm{K}=\mathrm{K}_{\mathrm{c}}$. Sin embargo, a menudo se observa que para que la fisura empiece a propagarse se debe aplicar una tensión no despreciable frente a la tensión de rotura inestable. Esto pone de manifiesto que en el momento en que se aplica la carga externa el primer término del segundo miembro de la ecuación [8] es inferior al valor de $\mathrm{K}_{\mathrm{c}}$ dado por la ecuación [3]. Una posible explicación de este comportamiento puede estar en que las tensiones residuales disminuyen cuando actúa una tensión externa que tiende a abrir la grieta [11]. En el caso presente, además existe la posibilidad de que las tensiones residuales sufran un cierto grado de relajación debido al mantenimiento del material a alta temperatura durante cierto tiempo previo al choque térmico. Por consiguiente, supondremos que el parámetro $\chi$ tiene un valor inferior a $\chi_{0}$. Entonces, la ecuación [8] se puede normalizar dividiendo por $\mathrm{K}_{\mathrm{c}}$ y se puede volver a escribir de forma mucho más sencilla mediante es:

$$
\frac{K}{K_{c}}=\mu z+\frac{1}{R z}
$$

donde

$$
\begin{aligned}
& \mu=\frac{\chi}{\chi_{0}} \\
& z=\sqrt{\frac{l_{0}}{l}}
\end{aligned}
$$

$\mathrm{y}$

$$
R=\frac{K_{c}}{Y \sigma \sqrt{l_{0}}}
$$

La fisura se extenderá durante el choque térmico si la

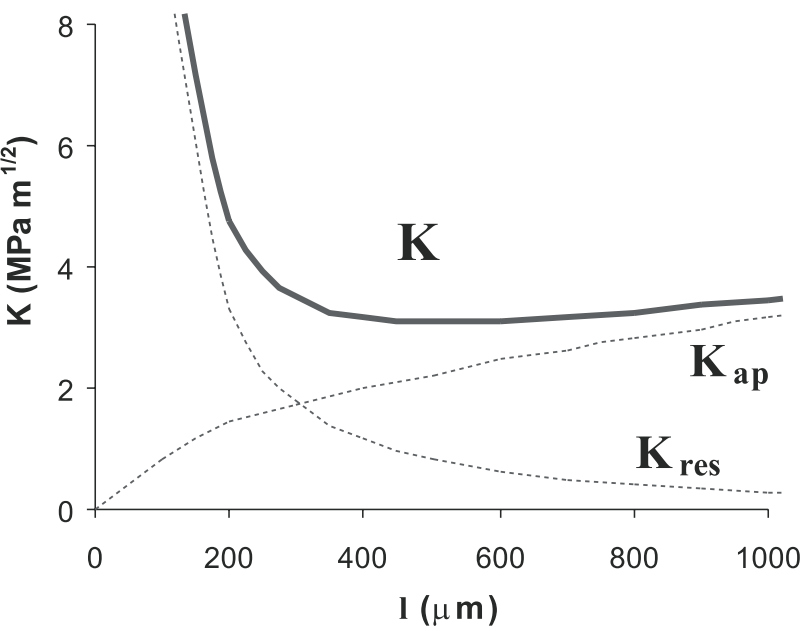

Figura 2. Factor de intensidad de tensiones total sobre una grieta Palmqvist en función de la longitud de la fisura. Se puede apreciar que K disminuye al propagarse la fisura hasta llegar a un valor mínimo a partir del cual aumenta.

tensión que se genera produce un factor de intensidad de tensiones suficientemente elevado para que junto al factor de intensidad de tensiones residuales se alcance un $\mathrm{K}$ total superior a $\mathrm{K}_{\mathrm{c}}$. Imponiendo $\mathrm{K} / \mathrm{K}_{\mathrm{c}}=1$ en la ecuación [9], se obtiene:

$$
\mu R z^{2}+1=z R
$$

Esta ecuación determina la extensión de la grieta para un determinado choque térmico siempre y cuando éste no sea suficientemente severo para producir la propagación inestable de la misma. Despejando R obtenemos una expresión únicamente en términos de $\mu$ y z. O sea,

$$
R=\frac{1}{z(1-\mu z)}
$$

La extensión será estable sí $\mathrm{dK} / \mathrm{dl}<0$, e inestable cuando $\mathrm{dK} / \mathrm{dl}>0$. La condición $\mathrm{dK} / \mathrm{dl}=0$ marca el límite de crecimiento estable que puede tener lugar durante el choque térmico. Derivando la ecuación [9] respecto a l, se obtiene:

$$
\frac{d\left(\frac{K}{K_{c}}\right)}{d l}=-\left(\frac{1}{2 l_{0}}\right) \frac{z}{R}\left(\mu R z^{2}-1\right)
$$

Imponiendo que dicha expresión sea igual a cero obtenemos una de las condiciones para el crecimiento inestable:

$$
\mu R z^{2}-1=0
$$

Ésta, junto a la ecuación [13], son las dos condiciones que deben cumplirse simultáneamente para que se produzca rotura inestable. La extensión estable de la fisura tendrá lugar hasta que $\mathrm{K}=\mathrm{K}$. Sumando y restando las ecuaciones [13] y [16] se puede determinar el máximo crecimiento estable de la grieta durante el choque térmico, así como el valor correspondiente de $\mathrm{R}$,

$$
z_{c r}=\frac{1}{2 \mu}
$$




$$
R_{c r}=4 \mu
$$

Estos valores son los críticos que pueden alcanzarse mediante choques térmicos que originen crecimiento estable de las fisuras. Valores inferiores causarán la rotura total del material. De esta última expresión se puede determinar la máxima tensión que puede soportar el material durante el cambio brusco de temperatura sin que se produzca la rotura total:

$$
\sigma_{c r}=\frac{K_{c}}{4 \mu Y \sqrt{l_{0}}}=\frac{\sigma_{0}}{4 \mu}
$$

donde $\sigma_{0}$ es la tensión de rotura en ausencia de tensiones residuales.

El parámetro R definido en la ecuación [12] representa el cociente entre la tenacidad de fractura y el factor de intensidad de tensiones inicial inducido por la tensión térmica. Es decir, R será mayor cuanto más tenaz sea el material y cuanto menor sea la tensión térmica y la longitud de grieta presente. Este parámetro puede utilizarse para la clasificación de la resistencia al choque térmico de diversos materiales frente a unas mismas condiciones de temple, o bien, utilizando un único material, estudiar la resistencia al choque térmico según la severidad del temple. La expresión deducida para $\mathrm{R}$, ecuación [14], depende únicamente de $z$, o sea, del cociente $1_{o} / 1$. Por tanto, para determinar $\mathrm{R}$ sólo es necesario medir la longitud de las grietas antes y después del choque térmico: no es necesario conocer el factor Y. Por el contrario, para determinar $\mathrm{R}_{\mathrm{m}}$ se requiere el conocimiento de la longitud de fisura inicial así como del factor $\mathrm{Y}$ :

$$
R_{m}=R^{2} Y^{2} l_{0}
$$

\section{PROCEDIMIENTO EXPERIMENTAL}

El material estudiado en el presente trabajo es una circona policristalina totalmente tetragonal (TZP) estabilizada con un 2.5\% molar de itria $\left(\mathrm{Y}_{2} \mathrm{O}_{3}\right)$. En la condición de recepción (condición $\mathrm{AR}$ ), presenta una microestructura de grano fino $(0.3 \mu \mathrm{m})$ con niveles elevados de resistencia a rotura (aprox. $1000 \mathrm{MPa}$ ) y valores de tenacidad de fractura comprendidos entre 4 y $5 \mathrm{MPa} \mathrm{m}^{1 / 2}$, ambas propiedades fueron medidas mediante flexión por tres puntos en probetas en forma de barras cilíndricas de $8 \mathrm{~mm}$ de diámetro con una distancia entre soportes de $40 \mathrm{~mm}$. En el caso de la tenacidad de fractura, se utilizó el método de "Indentation Fracture" el cual se basa en situar una fisura en la superficie sometida a tracción en el ensayo de flexión por tres puntos. Mediante diversos tratamientos térmicos a temperaturas alrededor de $1650{ }^{\circ} \mathrm{C}$ es posible aumentar la tenacidad de fractura del material hasta valores alrededor de $8 \mathrm{MPa} \mathrm{m}^{1 / 2}$. Esto se debe a una mayor transformabilidad de los granos tetragonales bajo la aplicación de esfuerzos así como a la aparición de fase cúbica, en cuyo interior pueden observarse precipitados tetragonales muy pequeños (12). Para realizar los diferentes ensayos de resistencia al choque térmico se cortaron pequeños discos de $4 \mathrm{~mm}$ de ancho, los cuales fueron desbastados y después pulidos con pastas de diamante de $30 \mu \mathrm{m}$ y $6 \mu \mathrm{m}$ de tamaño de partícula, con un acabado final de $3 \mu \mathrm{m}$. Posteriormente, las
TABLA I: PARÁMETROS EXPERIMENTALES Y DE RESISTENCIA AL CHOQUE TÉRMICO PARA Y-TZP SIN TRATAMIENTO TÉRMICO (AR). Y CON TRATAMIENTO TÉRMICO DE 2 HORAS A $1650{ }^{\circ} \mathrm{C},(2 \mathrm{H})$.

\begin{tabular}{|c|c|c|c|c|}
\hline \multicolumn{5}{|c|}{ MATERIAL AR } \\
\hline $\mathbf{P}(\mathbf{K g})$ & $\mathbf{l}_{\mathbf{0}}(\boldsymbol{\mu m})$ & $\mathbf{l}_{\mathbf{f}}(\mu \mathrm{m})$ & $\left.\Delta \mathbf{T} \mathbf{(}^{\mathbf{0}} \mathbf{C}\right)$ & $\mathbf{Z}$ \\
\hline 30 & 170 & 175 & 370 & 0.98 \\
30 & 162 & 177 & 380 & 0.96 \\
30 & 171 & 181 & 400 & 0.91 \\
30 & 164 & 179 & 405 & 0.97 \\
30 & 166 & 183 & 410 & 0.95 \\
30 & 170 & 204 & 435 & 0.91 \\
20 & 133 & 156 & 450 & 0.92 \\
20 & 135 & 170 & 475 & 0.89 \\
20 & 133 & 183 & 490 & 0.85 \\
20 & 135 & 183 & 510 & 0.85 \\
\hline
\end{tabular}

TABla II: PARÁmetros eXPerimentales Y DE ResistenCia AL CHOQUE TÉRMICO PARA Y-TZP CON TRATAMIENTO TÉRMICO DE 2 HORAS A 1650

\begin{tabular}{|c|c|c|c|c|}
\hline \multicolumn{5}{|c|}{ MATERIAL 2H } \\
\hline $\mathbf{P}(\mathbf{k g})$ & $\mathbf{l}_{\mathbf{0}}(\boldsymbol{\mu m})$ & $\mathbf{l}_{\mathbf{f}}(\mu \mathrm{m})$ & $\left.\Delta \mathbf{T} \mathbf{(}^{\mathbf{0}} \mathbf{C}\right)$ & $\mathbf{Z}$ \\
\hline 62.5 & 271 & 282 & 420 & 0.98 \\
62.5 & 309 & 360 & 455 & 0.93 \\
62.5 & 292 & 312 & 480 & 0.97 \\
62.5 & 286 & 306 & 480 & 0.96 \\
62.5 & 257 & 323 & 500 & 0.89 \\
62.5 & 276 & 367 & 520 & 0.87 \\
62.5 & 280 & 374 & 530 & 0.86 \\
30 & 148 & 273 & 540 & 0.74 \\
30 & 146 & 298 & 560 & 0.70 \\
20 & 100 & 245 & 600 & 0.64 \\
20 & 98 & 280 & 620 & 0.60 \\
\hline \multicolumn{5}{|r}{}
\end{tabular}

muestras fueron indentadas mediante un penetrador Vickers (Tabla I) y las longitudes de las fisuras producidas fueron medidas en un microscopio óptico utilizando el dispositivo de interferencia de Normarski. Finalmente las muestras fueron colocadas en un horno a diferentes temperaturas durante 15 minutos (Tabla II), y después sumergidas en aceite de silicona durante unos 20 minutos antes de medir el crecimiento de la fisura.

\section{RESULTADOS Y DISCUSIÓN}

Las fisuras de indentación realizadas son suficientemente largas para considerar que ya están completamente apantalladas. Es decir, suponemos que se ha alcanzado el "plateau" de la curva de energía específica de fractura frente a longitud de la fisura. Con esta hipótesis, el análisis anterior del proceso de choque térmico se puede aplicar aún cuando los materiales tengan una energía específica de fractura que varía con la extensión de la fisura. La diferencia entre las circonas AR y $2 \mathrm{H}$ reside en que para una misma carga de indentación, la longitud inicial de la fisura en la circona $2 \mathrm{H}$ es menor, ya que su tenacidad es mayor, $\mathrm{y}$, por tanto, el valor de $\mathrm{R}$ será mayor. Esto será así siempre y cuando sea cierta la hipótesis de que para un mismo gradiente de temperatura se produce la misma tensión térmica aún cuando la microestructura del material sea distinta (por ejemplo, en el tamaño y estructura cristalina de los granos). 


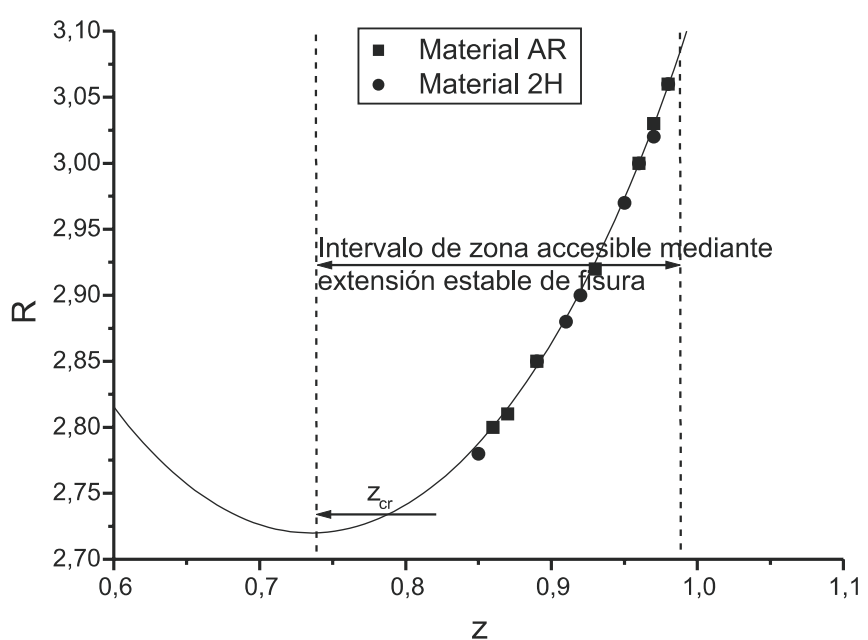

Figura 3. Representación de la función $\mathrm{R}$ y de los valores obtenidos experimentalmente para los distintos materiales, en función de la variable $\mathrm{z}$.

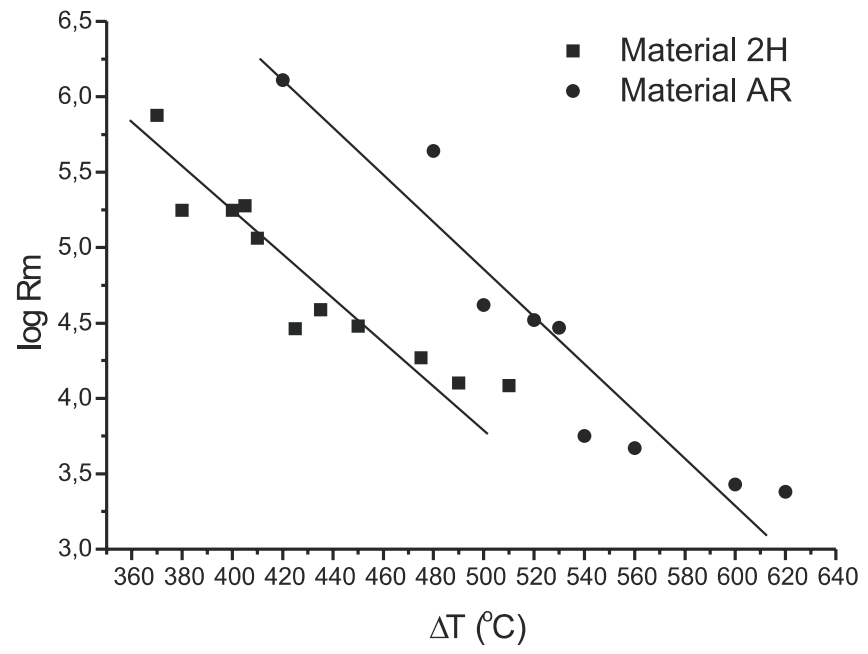

Figura 4. Representación gráfica del parámetro de Hasselman en función del gradiente de temperatura aplicado, para cada tipo de material.

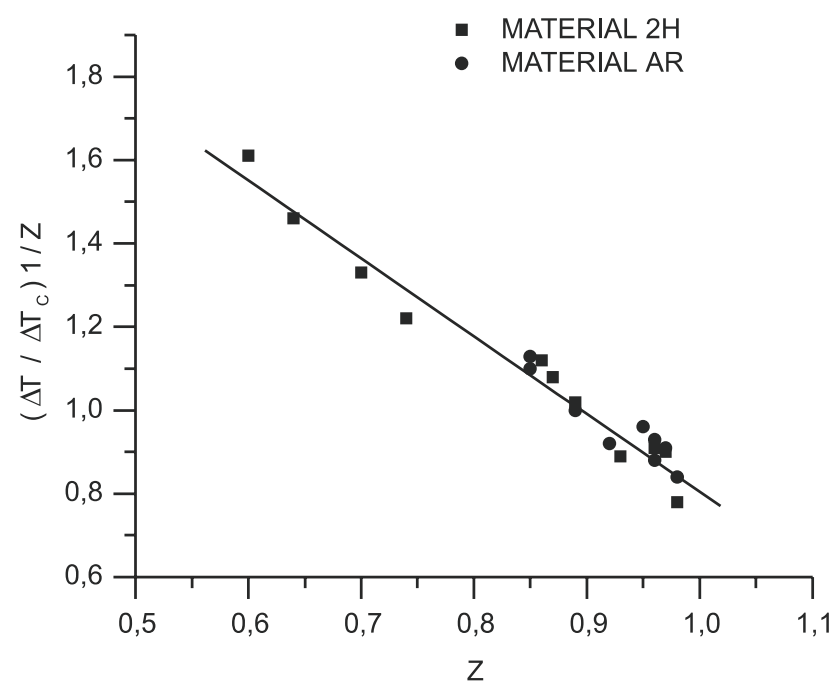

Figura 5. Representación de $(\Delta \mathrm{T} / \Delta \mathrm{T}) 1 / \mathrm{z}$ en función de z. De la pendiente se puede obtener el valor de $\mu$.
Los valores determinados de $\mathrm{R}$ y $\mathrm{R}_{\mathrm{m}}$ se han representado en las Figuras 3 y 4 . Es obvio que cuanto menor es la carga de indentación, la fisura creada es menor, y el material puede soportar mayores tensiones térmicas, o sea, mayores gradientes de temperatura. En el parámetro $\mathrm{R}_{\mathrm{m}}$ esto queda reflejado en la dependencia con $\sqrt{ } 1_{0}$. Por el contrario, el parámetro $\mathrm{R}$ no depende de la longitud inicial de la fisura, sino más bien del producto de la raíz cuadrada de ésta y la tensión térmica. Por tanto, depende solamente de z y de la reducción en las tensiones residuales a través del parámetro $\mu$.

Una observación importante es que las fisuras no se propagan hasta que se aplica un gradiente de temperatura relativamente elevado. Así, en la circona AR, se observa extensión de la fisura por primera vez cuando el gradiente de temperatura es cercano a $370^{\circ} \mathrm{C}$ para una carga de indentación de $30 \mathrm{Kg}$. La razón de este comportamiento probablemente es doble: i) es un hecho también observado en los ensayos de flexión que para empezar a propagar fisuras de indentación también es necesario aplicar una carga finita, lo cual se ha atribuido (11) a que las tensiones residuales disminuyen ligeramente al aplicar la carga externa en modo I; ii) al mantener la fisura a alta temperatura antes del choque térmico es posible cierto grado de relajación de las tensiones residuales. Ambos efectos se han considerado aquí introduciendo un parámetro $\chi$ en el término de las tensiones residuales menor que $\chi_{0}$ y suponiendo, por tanto, que $\mu$ es menor que 1 .

Si admitimos que la tensión máxima desarrollada en el disco durante el choque térmico es proporcional al cambio de temperatura, o sea, $\sigma \propto \Delta \mathrm{T}$, entonces se puede realizar una estimación del valor de $\mu$ considerando que para cualquier carga de indentación fija se cumple que

$$
\frac{\Delta T}{\Delta T_{c r}}=\frac{R_{c r}}{R}=4 \mu z(1-\mu z)
$$

El comportamiento experimental observado está en concordancia con esta ecuación, lo cual puede comprobarse representando $\Delta T / z \Delta T_{c r}$ frente a z. Según la ecuación [21], los puntos experimentales deben definir una recta de pendiente igual a $-4 \mu^{2}$. En la Fig. 5 se puede ver que todos los puntos experimentales obtenidos para las dos circonas definen la dependencia lineal esperada. A partir de la medida de la pendiente se obtiene $\mu \approx 0.68$, es decir, las tensiones residuales disminuyen alrededor de un $32 \%$ del valor original al formarse la huella de indentación.

Otro punto a considerar es que en el material AR no es posible encontrar un cambio de temperatura que produzca grietas cercanas a la longitud crítica que cabría esperar si no existiera una disminución en las tensiones residuales. En parte, la propuesta reducción en las tensiones residuales permite explicar este hecho. Simplemente, la ecuación [17] indica que $1=41_{0} \mu^{2}$, y por tanto, sí $\mu . \approx 0.68$, entonces la máxima longitud estable de la grieta debe ser inferior al doble de la longitud inicial, y no cuatro veces, tal como se esperaría si no hubiera disminución de las tensiones residuales. Sin embargo, aún con esta corrección las longitudes de grieta medidas en la condición AR son siempre bastante inferiores al valor crítico. Este efecto puede estar relacionado con la variación del factor de intensidad de tensiones total en puntos cercanos al mínimo. Se puede apreciar a partir de la ecuación [13] que la derivada $\mathrm{d}(\mathrm{K} / \mathrm{Kc}) / \mathrm{dl}$ disminuye al aumentar $1_{0}$, o sea, con la carga aplicada durante la indentación. En otras palabras, el valor total de $\mathrm{K}$ disminuye de forma más lenta con 1 si la carga de indentación es mayor. Por consiguiente, será más difícil encontrar grietas que con su crecimiento estable se puedan 
situar cercanas a la condición de inestabilidad ya que tan sólo muy pequeñas variaciones en el $\mathrm{K}$ total pueden producir la condición de inestabilidad.

Para la circona en la condición $2 \mathrm{H}$, su mayor tenacidad es la causa de que sea más resistente al choque térmico, es decir, soporta mayores gradientes de temperatura que los tolerados por el material AR (Ver tabla II). Sin embargo, todos los puntos obtenidos en la Fig.3 caen sobre la misma curva.

El hecho de que en la circona $2 \mathrm{H}$ sea posible medir longitudes de fisura mayores y más cercanas a la condición de inestabilidad se puede explicar si consideramos que cuanto mayor es la tenacidad, menor es la longitud de la grieta inicial, y por tanto, mayor es la pendiente de la curva de K total en función de z (ecuación [15]).

De la Tabla 2 se puede apreciar que en la circona en la condición $2 \mathrm{H}$ y para cargas de indentación pequeñas los valores de $\mathrm{z}$ medidos pueden llegar a ser incluso inferiores al valor crítico de z esperado de acuerdo a la ecuación [17]. La razón de esta discrepancia puede estar en que para cargas pequeñas de indentación, el tamaño de la grieta inicial (alrededor de $100 \mu \mathrm{m})$ puede ser demasiado pequeño para la validez de tanto la ecuación para las tensiones residuales como para la hipótesis de que la energía específica de fractura no dependa de la longitud de la grieta, ya que ésta puede que todavía no haya alcanzado el máximo de apantallamiento.

A partir del conocimiento del comportamiento de las fisuras estudiadas frente al choque térmico, es posible estimar el comportamiento de fisuras sin tensiones residuales pero con el mismo tipo y longitud de fisura inicial y con la misma geometría y las mismas condiciones de choque térmico. A partir de la expresión [19] que relaciona las tensiones de rotura para la cerámica con y sin tensiones residuales, se puede escribir:

$$
\Delta T_{\text {crit }}^{0}=4 \mu \Delta T_{\text {crit }}
$$

Es decir, el gradiente crítico cuando no hay tensiones residuales, $\Delta \mathrm{T}_{\text {crt }}{ }^{0}$, es igual a $4 \mu$ veces el valor del gradiente crítico en presencia de tensiones residuales. Puesto que la última ecuación se deduce directamente de la ecuación [19], aquí se ha analizado experimentalmente si ésta se cumple.

Para ello se procedió a realizar indentaciones en barras de Y-TZP en la condición AR y después se determinó su resistencia a flexión por tres puntos en tres conjuntos de probetas: a) probetas con un tratamiento a $1100{ }^{\circ} \mathrm{C}$ durante una hora para eliminar las tensiones residuales; $\mathrm{b}$ ) probetas con las tensiones residuales inducidas por indentación: c) probetas indentadas y después mantenidas a las temperaturas utilizadas para el choque térmico pero enfriadas lentamente en el horno. Tal como era de esperar, la resistencia a flexión de las probetas con tensiones residuales es inferior a la resistencia de las probetas sin tensiones residuales, siendo el valor del cociente fue de 0.61. Pero según la ecuación [19], el cociente:

$$
\frac{\sigma_{c r}}{\sigma_{0}}=\frac{1}{4 \mu}
$$

es igual a 0.37 , utilizando el valor de $\mu=0.68$ determinado a partir de la Fig. 5. En realidad no está justificado utilizar este valor de $\mu$ ya que éste fue encontrado en probetas expuestas a temperaturas de hasta $500{ }^{\circ} \mathrm{C}$ y la relajación de tensiones en este caso podría ser mayor que en el material sin ningún tipo de tratamiento térmico. Esta discrepancia no se puede asociar a propagación subcrítica de grieta durante el tiempo transcurrido después de la indentación, ya que para justificar las diferencias encontradas debería ocurrir una extensión muy grande de la fisura (del orden de la longitud de la fisura) y esto no se observa experimentalmente.

La resistencia a flexión de las probetas indentadas sin tratamiento posterior es inferior a la resistencia de probetas indentadas y con un tratamiento en el horno similar al realizado durante los ensayos de choque térmico (alrededor de $400-500{ }^{\circ} \mathrm{C}$ durante 15 minutos). El cociente entre ambas tensiones es de alrededor de 0.7. Es decir, durante este tratamiento previo al choque térmico se produce una disminución de las tensiones residuales de alrededor del $30 \%$.

\section{CONCLUSIONES}

Se ha analizado el comportamiento frente al choque térmico de policristales de circona tetragonal dopados con $2.5 \%$ molar de itria (Y-TZP) con dos niveles distintos de tenacidad y con fisuras de indentación del tipo Palmqvist. Las principales conclusiones del trabajo son:

Se propone la utilización de un parámetro de resistencia al choque térmico que puede determinarse experimentalmente midiendo únicamente la extensión de las fisuras por efecto de las tensiones térmicas generadas

El proceso de extensión de la fisura se cuantifica suponiendo que las tensiones residuales responsables de la propagación estable durante el choque térmico son inferiores a las presentes en el material inmediatamente después de aplicar la indentación, y se determina experimentalmente que esta reducción es de alrededor del $32 \%$.

\section{AGRADECIMIENTOS}

Este trabajo ha sido financiado parcialmente por el proyecto CICYT MAT97-0923. Igualmente, se agradece a la Generalitat de Catalunya la concesión de una beca F.I (D.C) y la ayuda recibida a través de la financiación a grupos consolidados 1997SGR00108.

\section{REFERENCIAS}

1. A.G. Evans, "Perspective on the development of high-toughness ceramics", J. Am. Ceram. Soc. 73 [2] 187-206 (1992).

2. B. Lawn, "Fracture mechanics of brittle solids", Cambridge University Press, U.K. (1995).

3. W.D. Kingery, "Factors affecting thermal stress resistance of ceramic materials", J. Am. Ceram.Soc. 38, 3-15 (1955)

4. D.P.H. Hasselman, "Elastic energy as design criteria for thermal shock", J. Am. Ceram.. Soc., 46 [11535-40 (1963).

5. D.P.H. Hasselman, “Thermal stress resistance parameters for brittle refractory ceramics: A compendium", Am. Ceram. Soc. Bull., 49 [12] 1033-37 (1970).

6. F. Tancret y F. Ostertock, "The Vickers indentation technique used to evaluate thermal shock resistance of brittle materials", Scripta Materialia, 37 [4] 443-47 (1997).

7. D.K. Shetty, A.R. Rosenfield, y W.H. Duckworth, "Indenter flaw geometry and fracture toughness estimates for a glass-ceramic" , J. Am. Ceram. Soc., 68 [10] C-282-C-284 (1985).

8. K. Nihihara, R. Marena y D.P.H. Hasselman: J. Mater. Sci. Lett.. 1982.1.1316.

9. M.T. Laugier, "Palmqvist crack extension and the center-loaded penny crack analogy", J. Am. Ceram. Soc., 68 [2] C51-C52 (1985).

10. J.C. Newman y I.S. Raju, "An empirical stress-intensity factor equation for surfarce crack", Eng. Frac. Mec., 15 [1-2] 185-92 (1981).

11. T. Fett, "An analysis of the residual intensity factor of Vickers indentation cracks", Eng. Frac. Mec., 52 [4] 773-76 (1995).

12. D. Casellas, L. Llanes y M. Anglada, "Fracture resistance of heat treated Y-TZP", in Ceramics gettings into the 2000 's, Part A, Edited by Vincenzini, 13 705-12 (1998). 